\title{
Numerical simulation of rock fracture using three-dimensional extended discrete element method
}

\author{
Yuya Matsuda $^{1}$ and Yasuyuki Iwase ${ }^{2}$ \\ ${ }^{1}$ Department of Earth and Planetary Systems Science, Graduate School of Science, Hiroshima University, \\ 1-3-1 Kagamiyama, Higashi-Hiroshima 739-8526, Japan \\ ${ }^{2}$ Department of Earth and Ocean Sciences, School of Applied Sciences, National Defense Academy, \\ 1-10-20 Hashirimizu, Yokosuka 239-8686, Japan
}

(Received May 7, 2001; Revised February 4, 2002; Accepted February 4, 2002)

\begin{abstract}
We perform three-dimensional numerical simulation of rock fracturing under uniaxial compression by extending the discrete element method (DEM). Rock sample is modeled as an assemblage of about four thousand spheres having the same radius. Each element satisfies equations of motion for both translation and rotation. In extension of the DEM, we assume cohesion between elements and constrained rotation of the elements; these assumptions are required to treat the continuum by the DEM. We study two cases of uniaxial compression tests: A homogeneous sample having an equal cohesion force between elements and heterogeneous sample having weak parts of cohesion in one percent of the total number of the bonds of elements. We present the detail of fracturing process of model rock samples and obtain stress-strain curve for each case. The homogeneous sample shows a cone-shaped fault system, whereas the heterogeneous sample shows complex fault system consisting of major and sub faults. We find that the inner stress and rotation of elements show the negative correlation during fracturing process. The results are in good agreement with both experimental and theoretical results.
\end{abstract}

\section{Introduction}

Earth and the other terrestrial planets mainly consist of rocks. Physical properties, elastic-plastic deformations and fracturing, of rocks provide a clue to understand the formation and evolution of the planets. Fracturing of rock is considered to be one of the most important processes for planetary dynamics and related to earthquake, orogeny, plate motion, and so forth. Numerous theoretical studies in the view of dislocation, linear-elastic fracture mechanics (e.g., Knott, 1973; Weertman, 1996) and laboratory experiments (e.g., Vutukuri et al., 1974; Paterson, 1978) have been reported fracturing behavior of rocks under stress. To the contrary, fracturing mechanisms of the rocks have not yet been well clarified by either experimental or theoretical studies for the following characteristics of the fracturing process: (1) irreversible, nonlinear, and nonequilibrium; (2) various spatial scales; (3) complex effect of inhomogeneity within the medium (e.g., Scholz, 1990).

Recent remarkable development in computer technology provides methods for studying rock fracturing by numerical simulation, which can inherently control the entire mechanical properties. Numerical simulations for continuum medium theory enable to predict locations of fracture (i.e., macroscopic approach). However, their demerit is the difficulty in solving the discontinuities such as faults requiring the utilization of complex functions (e.g., Bird and Kong, 1994; Wu et al., 1998). At a microscopic level, for example, by molecular dynamics (MD), such problem may be completely solved in principle (e.g., Holland and Marder, 1998), though a huge amount of computer resources is needed for this approach.

As one of the mesoscopic approaches, the discrete (distinct) element method (DEM) was developed by Cundall and Strack (1979). The fundamental concept of this method is simple: An object is considered to consist of elements, a discrete unit of material, and the equation of motion for each element is solved. The DEM has been employed in the analyses of geological structures, the movement of soil, and others (Donzé et al., 1994; Iwashita and Oda, 1998; Mora and Place, 1998; Place and Mora, 1999) to show good agreements with observations and experimental results. Most of those simulations are, however, required to introduce complex mathematics due to the nature of elements or mechanisms of phenomena (Iwashita and Oda, 1998; Mora and Place, 1998; Place and Mora, 1999), furthermore are done under two-dimensional condition.

In this study, we extend the DEM under three-dimensional condition in order to model the fracturing processes of rock sample under uniaxial stress. The main advantage of numerical simulation is to observe the details of fracturing zone during rock deformation, which cannot be realized in laboratory experiments. On the basis of numerical simulation, we investigate the effect of weak part inside the rock on fracturing. Evolution of cracks and stress-strain relationship are also studied.

Copy right(C) The Society of Geomagnetism and Earth, Planetary and Space Sciences (SGEPSS); The Seismological Society of Japan; The Volcanological Society of Japan; The Geodetic Society of Japan; The Japanese Society for Planetary Sciences. 
Table 1. List of symbols.

\begin{tabular}{|c|c|}
\hline Variable & Meanings \\
\hline$A$ & contact damping coefficient \\
\hline$a_{n}, a_{s}$ & normal and shear viscosities \\
\hline$B$ & rotational contact damping coefficient \\
\hline$b$ & rotational viscosity \\
\hline$c$ & cohesion between two elements \\
\hline$d^{\prime}, d$ & relative displacement at $t=t-\Delta t$ and $t=t$ \\
\hline $\boldsymbol{F}$ & contact force \\
\hline$f_{n}, f_{s}$ & normal and shear forces \\
\hline$h_{n}, h_{s}, h_{r}$ & normal, shear and rotational damping factors \\
\hline$I_{0}$ & moment of inertia of an element \\
\hline$k_{n}, k_{s}, k_{r}$ & normal, shear and rotational elastic constants \\
\hline$M$ & moment of the force \\
\hline$m_{0}$ & mass of an element \\
\hline$r_{0}$ & radius of an element \\
\hline $\boldsymbol{u}$ & the position of center of element \\
\hline$\Delta f_{n}, \Delta f_{s}, \Delta f_{r}$ & increases of normal, shear and rotational forces during $\Delta t$ \\
\hline$\Delta n, \Delta s$ & increases of normal and shear relative displacements during $\Delta t$ \\
\hline$\Delta t$ & time step \\
\hline$\Delta \phi$ & increase of rotation of element during $\Delta t$ \\
\hline$\Delta \theta$ & increase of relative rotation between the elements during $\Delta t$ \\
\hline$\mu$ & coefficient of friction for one element \\
\hline$\phi$ & rotation angle of the element \\
\hline
\end{tabular}

\section{Numerical Method}

The DEM has been in fact used for studies of discontinuum media, such as granular soils (Cundall and Strack, 1979), i.e., a sand corresponds to an element. In order to consider the deformation and fracturing of rock, however, the DEM must be modified to be applicable for both continuum and discontinuum media. We assume presence and absence of cohesion force between neighboring elements respectively for continuum and discontinuum. The evolutions of cracks and faults are interpreted as the movement of a number of elements, if the rock is modeled as an assemblage of many elements. The outline of the DEM and our modification are described below.

Visco-elastic spherical elements with a radius, $r_{0}$, mass, $m_{0}$, and moment of inertia, $I_{0}\left(r_{0}, m_{0}\right.$ and $I_{0}$ are constants) under consideration are assumed to be Voigt-Kelvin material. Thus, each element satisfies the following equations of motion;

$$
\left\{\begin{array}{l}
m_{0} \ddot{\boldsymbol{u}}_{i}+A_{i} \dot{\boldsymbol{u}}_{i}+\boldsymbol{F}_{i}=0, \\
I_{0} \ddot{\boldsymbol{\phi}}_{i}+B_{i} \dot{\boldsymbol{\phi}}_{i}+\boldsymbol{M}_{i}=0,
\end{array}\right.
$$

where the subscript $i$ refers to the $i$-th element; the number of dots stands for the order of differentiation with respect to time; $\boldsymbol{F}_{i}$ and $\boldsymbol{M}_{i}$ are, respectively, sum of the contact forces and the moment of the force acting on the $i$-th element from the neighboring elements; $A_{i}$ and $B_{i}$ are the contact damping coefficients; $\boldsymbol{u}_{i}$ is the position, and $\boldsymbol{\phi}_{i}$ is the rotation angle of the element $i$. Symbols used in this paper are summarized in Table 1. The second and third terms in Eq. (1) represent the viscous and the elastic or frictional forces, respectively. The solution given by numerical time integration provides the position and rotation angle of the element.

When elements $i$ and $j$ are connected or contacted, the increase in the interaction forces between the elements $i$ and $j$ during time $t-\Delta t$ and $t$ is calculated by the relative displacements between each other (Fig. 1). The normal $\left(\Delta \boldsymbol{n}_{(i j)}\right)$ and shear $\left(\Delta \boldsymbol{s}_{(i j)}\right)$ components of the relative displacements may be given by

$$
\begin{aligned}
& \Delta \boldsymbol{n}_{(i j)}=\left\{\left|\boldsymbol{d}_{i j}\right|-\frac{\boldsymbol{d}_{i j}^{\prime} \cdot \boldsymbol{d}_{i j}}{\left|\boldsymbol{d}_{i j}\right|}\right\} \frac{\boldsymbol{d}_{i j}}{\left|\boldsymbol{d}_{i j}\right|}, \\
& \Delta \boldsymbol{s}_{(i j)}=\boldsymbol{d}_{i j}-\boldsymbol{d}_{i j}^{\prime}-\Delta \boldsymbol{n}_{(i j)},
\end{aligned}
$$

where

$$
\begin{aligned}
& \boldsymbol{d}_{i j}^{\prime}=\boldsymbol{u}_{j}(t-\Delta t)-\boldsymbol{u}_{i}(t-\Delta t), \\
& \boldsymbol{d}_{i j}=\boldsymbol{u}_{j}(t)-\boldsymbol{u}_{i}(t) .
\end{aligned}
$$

The relative rotation between the elements $i$ and $j$ during $\Delta t, \Delta \boldsymbol{\theta}_{(i j)}$, is written by

$$
\Delta \boldsymbol{\theta}_{(i j)}=\Delta \boldsymbol{\phi}_{j}-\Delta \boldsymbol{\phi}_{i} .
$$

Using the equations above, the increases of the elastic force on the element $i$ from the element $j$ is expressed by

$$
\Delta \boldsymbol{f}_{n_{(i j)}}=k_{n} \Delta \boldsymbol{n}_{(i j)}
$$




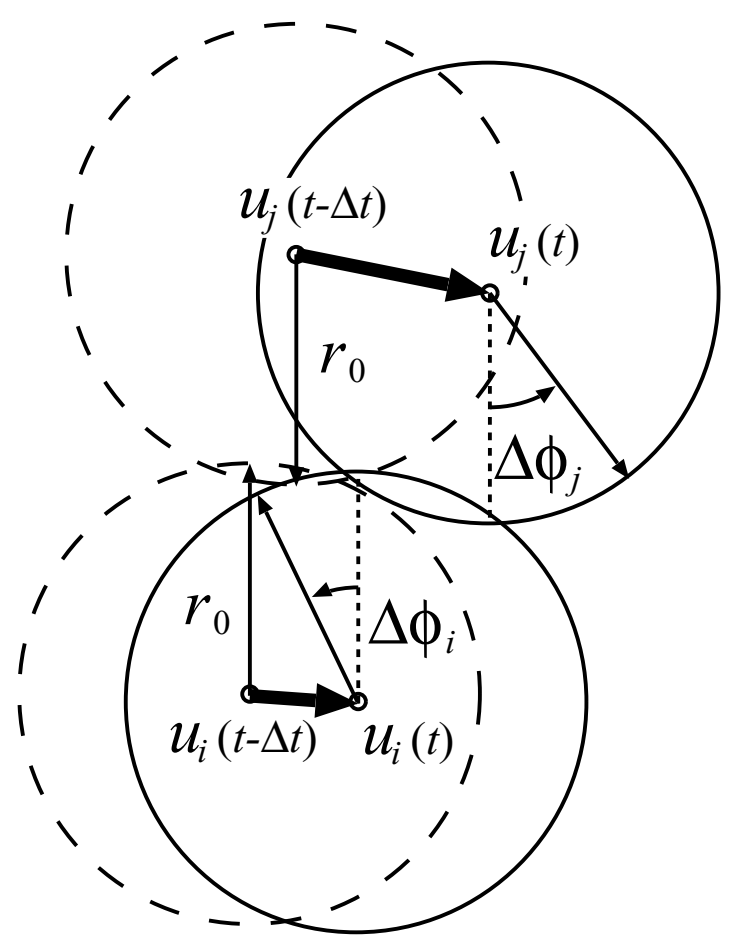

Fig. 1. A schematic concerning displacements of elements. Dashed and solid circles indicate the elements at $t=t-\Delta t$ and $t=t$, respectively. The positions of the center of elements $i$ and $j$ at $t=t-\Delta t$ are $\boldsymbol{u}_{i}(t-\Delta t)$ and $\boldsymbol{u}_{j}(t-\Delta t)$, and those at $t=t$ are $\boldsymbol{u}_{i}(t)$ and $\boldsymbol{u}_{j}(t)$, respectively. Each element rotates the angle $\Delta \boldsymbol{\phi}_{i}$ and $\Delta \boldsymbol{\phi}_{j}$ during $\Delta t$.

$$
\begin{aligned}
& \Delta \boldsymbol{f}_{s_{(i j)}}=k_{s} \Delta \boldsymbol{s}_{(i j)}, \\
& \Delta \boldsymbol{f}_{r_{(i j)}}=k_{r} \Delta \boldsymbol{\theta}_{(i j)},
\end{aligned}
$$

where the subscripts $n, s$ and $r$ refer to normal, tangential and rotational directions, respectively, $k_{n}, k_{s}$ and $k_{r}$ are the elastic constants. In this study, we assume $k_{n}=k_{s}$ and $k_{r}=2 r_{0} k_{s}$ (constant). Provided that elements are connected to each other, no rotation occurs $\left(\Delta \boldsymbol{\theta}_{(i j)}=0\right)$.

Thus, the normal and tangential forces at $t$ are given by

$$
\begin{aligned}
& \boldsymbol{f}_{n_{(i j)}}(t)=\boldsymbol{f}_{n_{(i j)}}(t-\Delta t)+\Delta \boldsymbol{f}_{n_{(i j)}}, \\
& \boldsymbol{f}_{s_{(i j)}}(t)=\boldsymbol{f}_{s_{(i j)}}(t-\Delta t)+\Delta \boldsymbol{f}_{s_{(i j)}}+\Delta \boldsymbol{f}_{r_{(i j)}} / r_{0} .
\end{aligned}
$$

The failure of each connection is assumed to be determined by the Coulomb-Mohr's criterion given by

$$
f_{s_{(i j)}} \geq c+\mu f_{n_{(i j)}}
$$

where $f_{s_{(i j)}}$ and $f_{n_{(i j)}}$ is the absolute values of $\boldsymbol{f}_{s_{(i j)}}$ and $\boldsymbol{f}_{n_{(i j)}}$ (compression; positive and extension; negative), respectively; $c$ is the cohesion, and $\mu$ is the coefficient of friction. Once Eq. (12) is satisfied, the connection between the elements $i$ and $j$ is lost and will never be recovered.

When detached elements come into contact, a frictional force is also taken into account. The frictional force appears only when the predicted contact forces, without friction, calculated by Eqs. (10) and (11) satisfy the following inequality,

$$
f_{S_{(i j)}} \geq \mu f_{n_{(i j)}} .
$$

Then, the elements are slipped and the virtual tangential force is restricted to

$$
\boldsymbol{f}_{s_{(i j)}} \leftarrow \mu f_{n_{(i j)}} \frac{\boldsymbol{f}_{s_{(i j)}}}{f_{S_{(i j)}}} .
$$

Otherwise $\boldsymbol{f}_{s_{(i j)}}$ remains to be equal to that in Eq. (11).

In our work, the linear viscous terms are present, only if there is any interaction among the elements. The viscous terms represent the resistance force against displacement, making the simulation less microscopic and closer to quasistatic process, which is appropriate for tectonic modeling. The dynamic viscosities are assumed as

$$
\begin{aligned}
a_{n} & =2 h_{n} \sqrt{m_{0} k_{n}}, \\
a_{s} & =2 h_{s} \sqrt{m_{0} k_{s}}, \\
b & =2 h_{r} \sqrt{m_{0} r_{0} k_{r} / 2},
\end{aligned}
$$

where $h_{x}(x=n, s, r)$ is the damping factor $\left(0 \leq h_{x} \leq 1\right)$. When $h_{x}$ is unity, critical damping takes place.

Table 2. Parameters used in the simulation.

\begin{tabular}{ll}
\multicolumn{1}{c}{ Parameter } & \multicolumn{1}{c}{ Values } \\
\hline Number of elements & 4163 \\
Radius of an element $\left(r_{0}\right)$ & $0.05(\mathrm{~m})$ \\
Mass of an element $\left(m_{0}\right)$ & $1.12(\mathrm{~kg})$ \\
Elastic constant $\left(k_{s}\right)$ & $10^{6}\left(\mathrm{~kg} \mathrm{~s}^{-2}\right)$ \\
Young's modulus & $2.0 \times 10^{7}\left(\mathrm{~kg} \mathrm{~m}^{-1} \mathrm{~s}^{-2}\right)$ \\
Time step $(\Delta t)$ & $10^{-4}(\mathrm{~s})$ \\
Strain rate & $10^{-3}\left(\mathrm{~s}^{-1}\right)$ \\
Cohesion $(c)$ & $1.27 \times 10^{2}\left(\mathrm{~kg} \mathrm{~m} \mathrm{~s}^{-2}\right)$ \\
Coefficient of friction $(\mu)$ & 0.6 \\
Damping factor $\left(h_{n}, h_{s}, h_{r}\right)$ & 0.2 \\
\hline
\end{tabular}

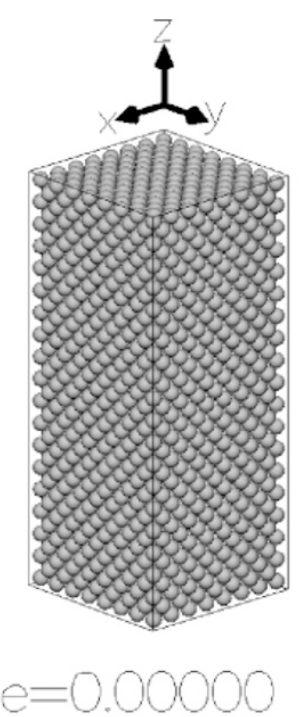

Fig. 2. Initial configuration of elements. The rectangular parallelepiped represents initial shape of model rock sample. The $e$ represents strain along vertical axis. Arrows indicate the direction of coordinates. 
(a)

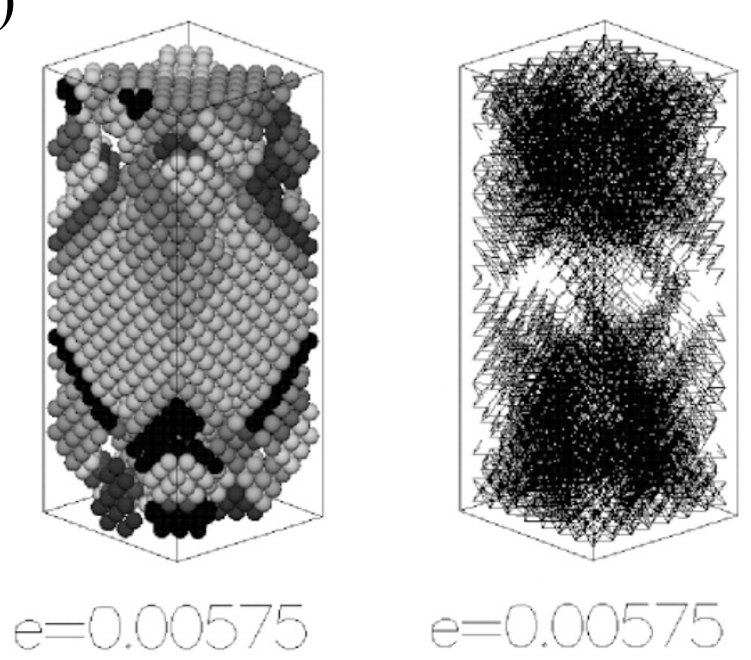

Fig. 3(a). Configuration of elements (left) and cracks (right) for case A after the fracturing ( $e=0.00575$ ). In the left figure, the dislocated units consisting of more than four connected elements are shown by different color tones. In the right figure, the disconnection lines are drawn by lines connected the center of elements when elements are disconnected. Frame boxes indicate the initial shape of model rock.

(b)

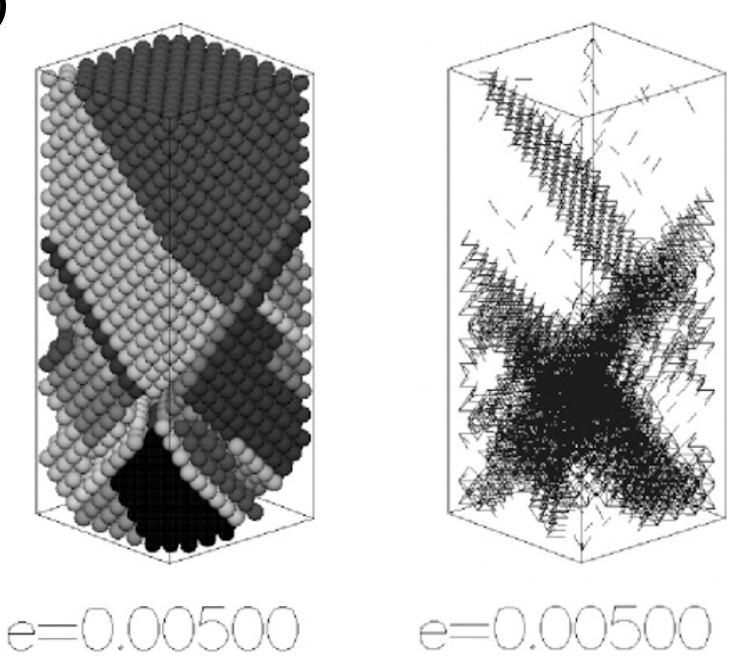

Fig. 3(b). Configuration of elements (left) and cracks (right) for case B after the fracturing $(e=0.00500)$.

The total elastic or frictional force and the total moment acting on $i$-th element are respectively

$$
\begin{aligned}
\boldsymbol{F}_{i} & =\sum_{j, j \neq i}\left(\boldsymbol{f}_{n_{(i j)}}+\boldsymbol{f}_{S_{(i j)}}\right), \\
\boldsymbol{M}_{i} & =\sum_{j, j \neq i} r_{0} \frac{\boldsymbol{d}_{i j}}{\left|\boldsymbol{d}_{i j}\right|} \times \boldsymbol{f}_{S_{(i j)}},
\end{aligned}
$$

and the viscous terms are given by

$$
\begin{aligned}
A_{i} \dot{\boldsymbol{u}}_{i} & =\sum_{j, j \neq i}\left(a_{n} \Delta \boldsymbol{n}_{(i j)}+a_{s} \Delta \boldsymbol{s}_{(i j)}+b \Delta \boldsymbol{\theta}_{(i j)}\right) / \Delta t, \\
B_{i} \dot{\boldsymbol{\phi}}_{i} & =\sum_{j, j \neq i} b r_{0} \Delta \boldsymbol{\theta}_{(i j)} / \Delta t
\end{aligned}
$$

Above calculations are performed for each element in turn. The Verlet algorithm (Tuckerman et al., 1992) is used for time integration in Eq. (1). The time step, $\Delta t$, is set to be less than $2 \sqrt{m_{0} / k_{s}}$, to prevent generation of shock wave.

\section{Model}

Before applying our code to complex geological structure, uniaxial compression of rock is simulated in this study. The uniaxial compression experiment is one of the basic tests of rock deformation and fracture (Paterson, 1978) and the numerical simulation should be compared with the experimental results. Parameters and physical properties used in this study are listed in Table 2.

Two cases (cases A and B) are studied. In both cases the model rock consists of about four thousands spherical elements (Fig. 2). The shape of model rock sample is a rectangular parallelepiped with an aspect ratio of $1: 1: 2.5$, in which the elements are configured with face-centered cubic $(f c c)$. 

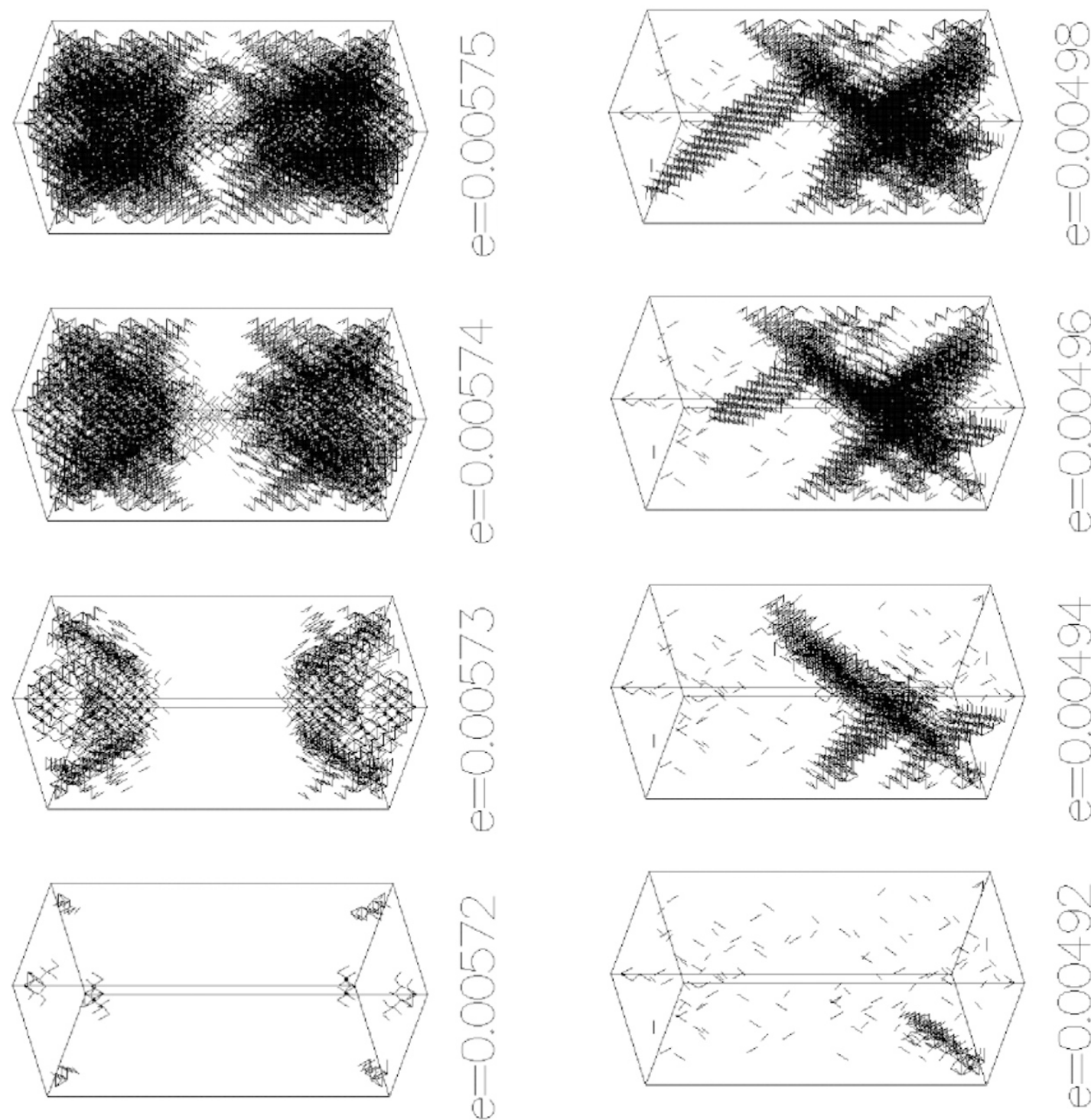

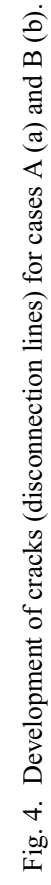
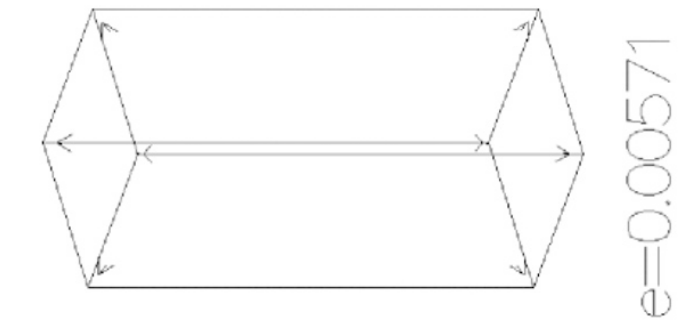

(્)

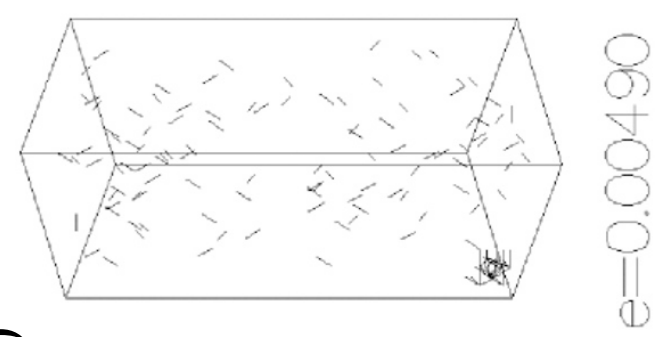

$\hat{e}$ 
(a)
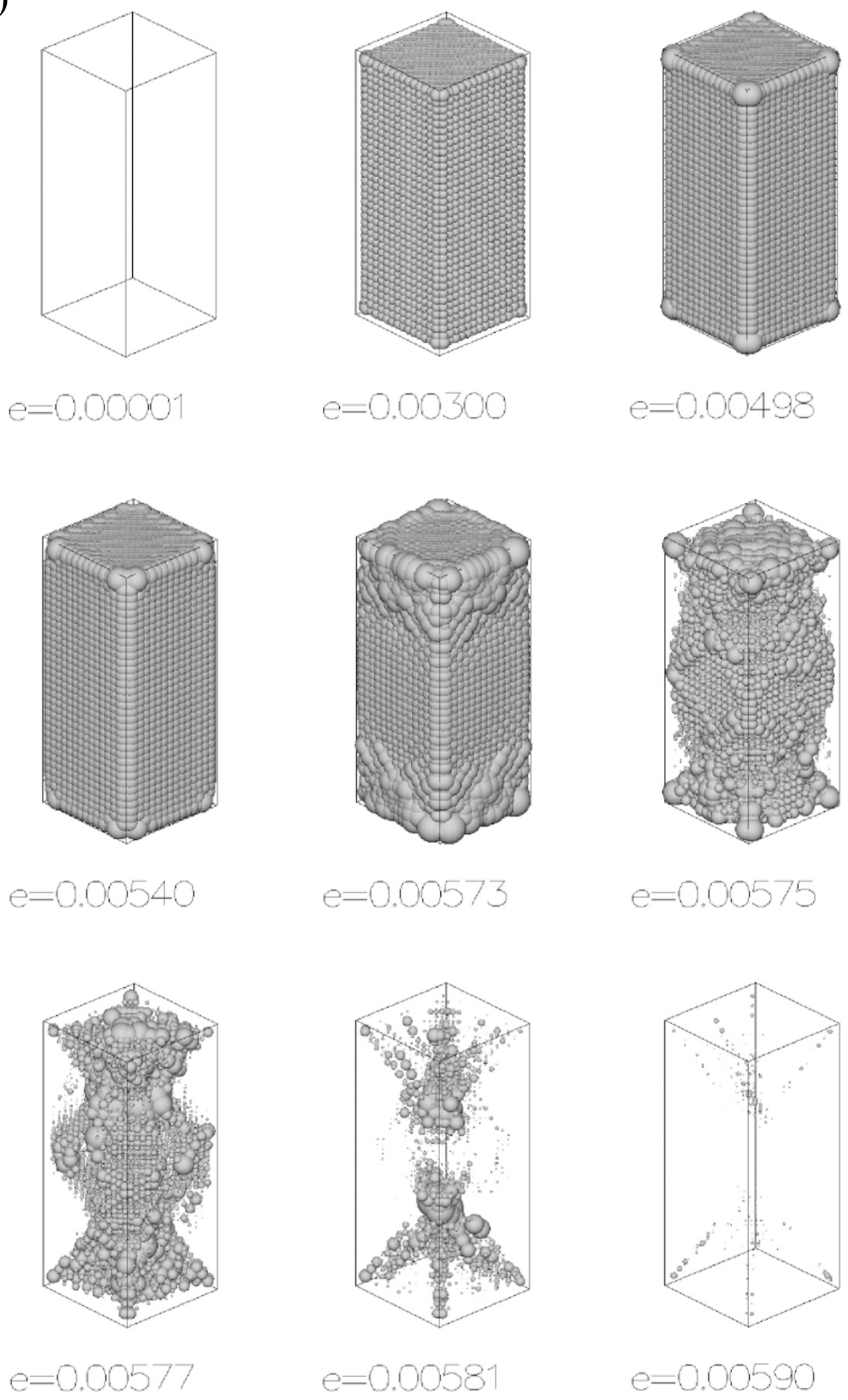

Fig. 5. Development of stress sphere for case A (a) and B (b). The radii of sphere indicate the size of normal force $\left(f_{n}\right)$ for each element in contact or connection.

For the convenience of description, we set the $x$-, $y$-, and $z$ axes parallel to edges of the sample, as shown in Fig. 2. We assume that the neighboring elements are initially connected and all the elements have zero rotational angle $\left(\boldsymbol{\phi}_{i}=0\right)$.
When compressing the rock, the walls are set on both top and bottom surfaces of model rock sample and the coefficient of friction of the walls is the same for that of the element. The side surfaces are stress-free. In case B, to avoid 
(b)
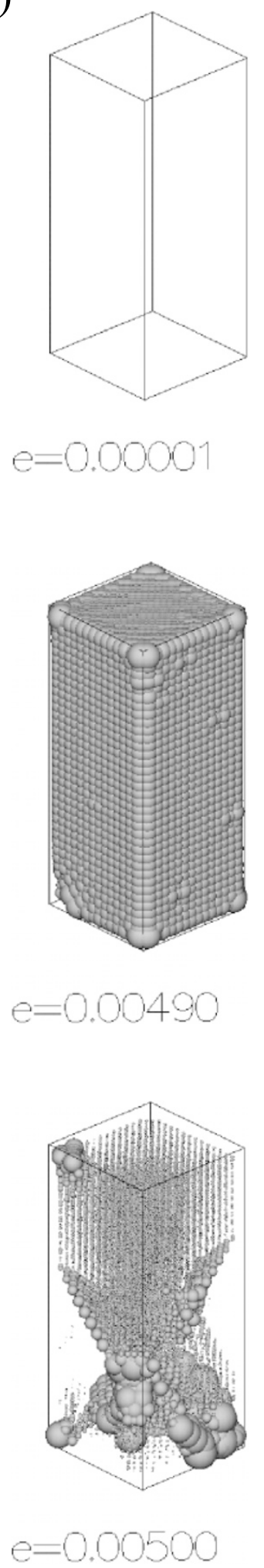
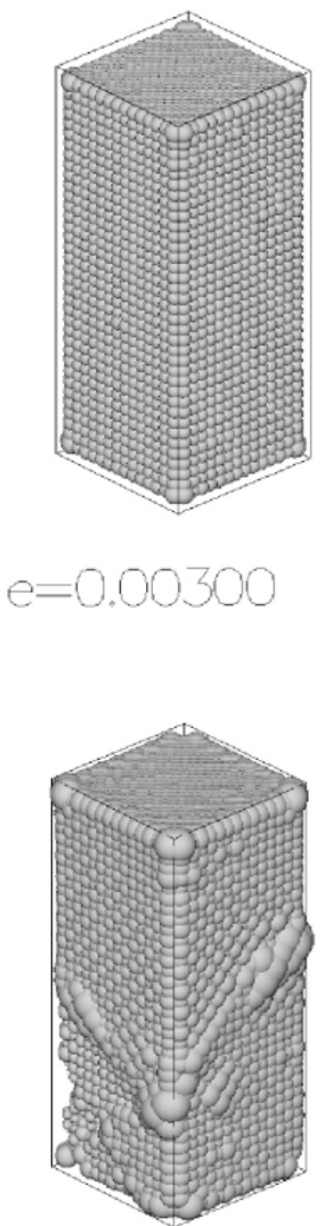

$e=0.00494$
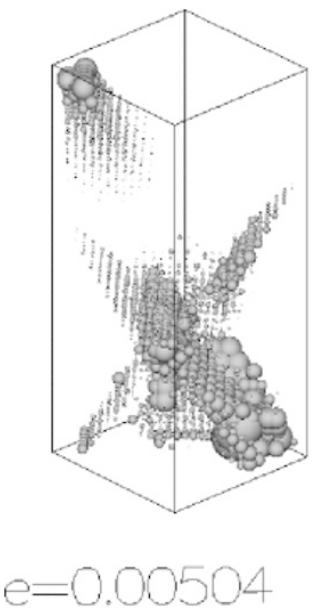
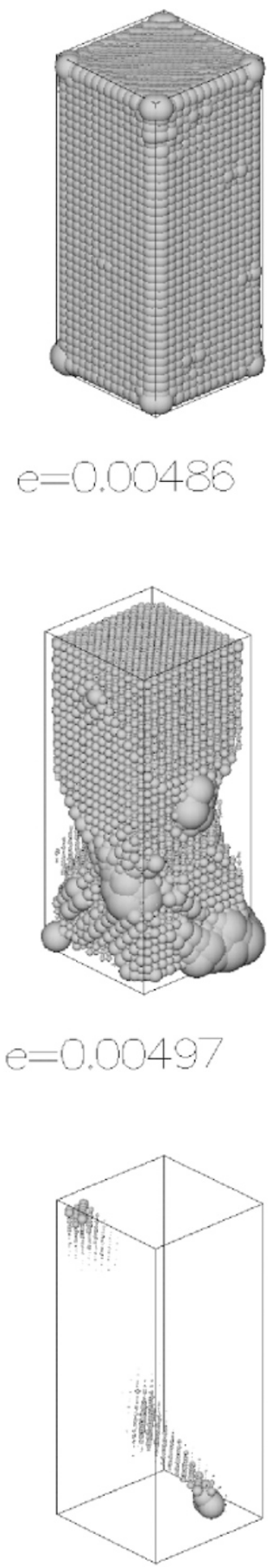

$e=0.00600$

Fig. 5. (continued).

the fracture due to the symmetry of the $f c c$ configuration of elements, the cohesion, $c$, of random selected connections of elements (one percent of the total connections) are reduced by a factor of $0-1$, whereas homogeneous cohesion is used for case A. The model rocks are pressed with a con- stant strain rate $\left(10^{-3} \mathrm{~s}^{-1}\right)$, by moving both upper and lower walls along the $z$-axis. The compression starts at $t=0 \mathrm{~s}$; at this time, the vertical axial strain is zero $(e=0)$. Calculations are continued until $e=0.010(t=10 \mathrm{~s})$; the time when the fracture process has finished. 
(a)
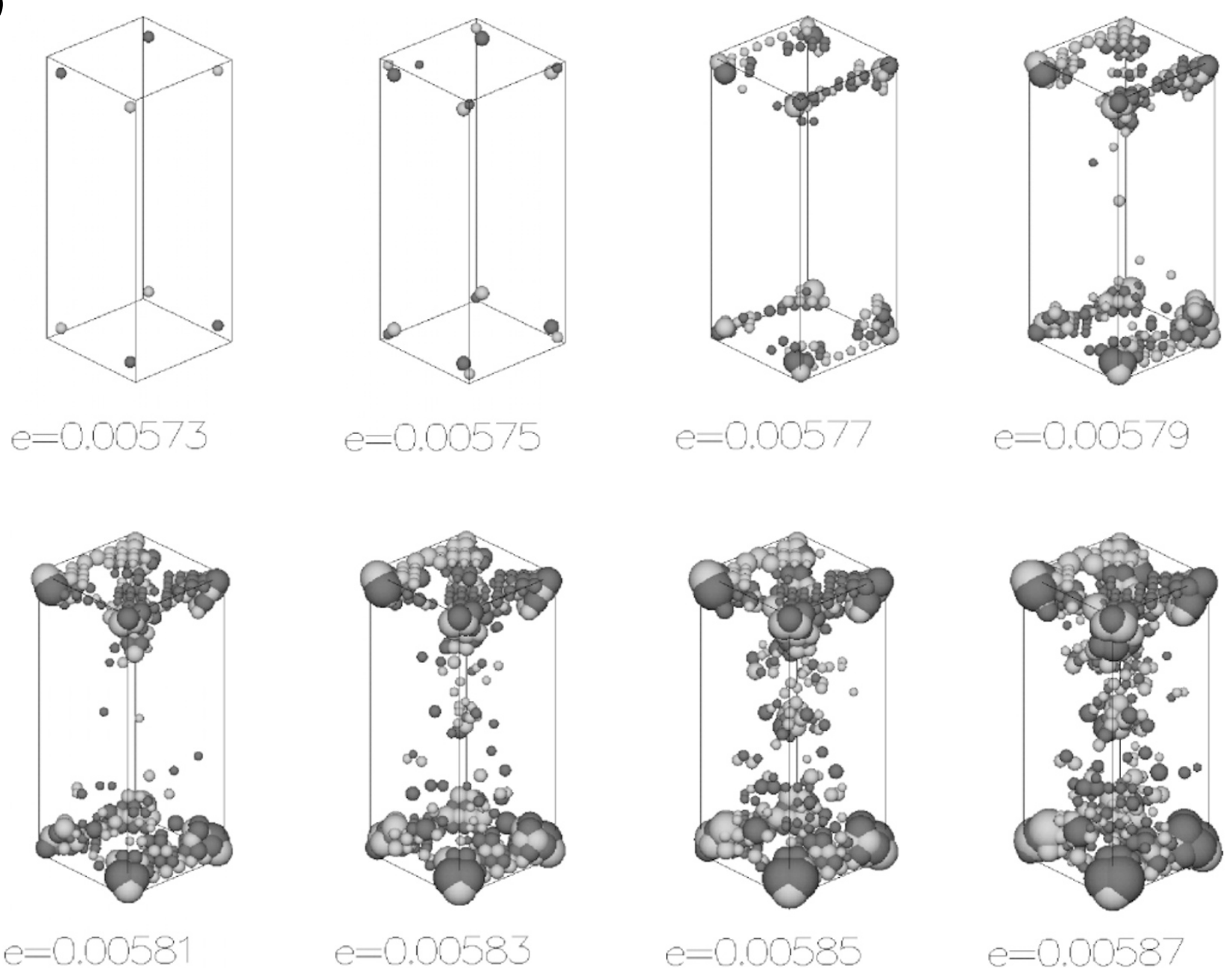

Fig. 6. Development of rotation sphere of case A (a) and B (b). The radii of sphere indicate the angle of rotation ( $\phi)$ for each element on $x$-axis. Light gray denotes clockwise rotation and dark gray denotes counterclockwise rotation.

\section{Results and Discussion \\ 4.1 Fracturing process}

Figures 3(a) and (b) show the configuration of elements after fracture for case A $(e=0.00575 ; t=5.75 \mathrm{~s})$ and for case $\mathrm{B}(e=0.00500 ; t=5.00 \mathrm{~s})$, respectively. The dislocated units consisting of more than four connected elements are shown by different color tones (left) and the disconnection lines (right); the disconnection lines tie between the centers of the two elements in disconnection. The disconnection line may be interpreted as cracking or microfailure. The fracture zone, that is, the zone where the distribution of the disconnection lines is dense, appears cone-shaped for the case A and oblique for the case B. Note that downward dropping of fragments is not observed because of zero gravity assumed.

Evolution of disconnection lines is shown as a function of time in Figs. 4(a) and (b) for cases A and B, respectively. For case A, cracking begins at the edges of both the upper and lower corners and propagates toward the center of the rock sample. This process occurs within a short time $(<0.05 \mathrm{~s})$ and the corresponding strain, $e$, is $0.00571-0.00575$. For case $\mathrm{B}$, although the failure first occurs at the connections where the cohesion is reduced, the major fracturing initiates at a corner, propagates toward the central part, and results in the coalescences of cracks. As shown in Figs. 3(a) and 4(a), in the homogeneous sample (case A), the fracture zone becomes cone-shaped which is consistent with the theoretically predicted weak zone of rocks (Bordia, 1971). In contrast to the case A, a complex system of fracturing surface consisting of major faults and sub faults appears in the heterogeneous sample (case B; Figs. 3(b) and 4(b)). Both patterns of fracturing surfaces are observed in laboratory experiments (Lama, 1966; Paterson, 1978), suggesting that the fracture pattern is controlled by the distribution of strength inside the rock.

\subsection{Stress distribution}

The pattern of fracturing surfaces has been considered to be determined by the distribution of microfailure and localization of stress concentration around cracks (Paterson, 1978). Stress distribution inside the rock are shown in Figs. 5(a) and (b). The size of spheres shown in Figs. 5(a) and (b) is proportional to the magnitude of normal stress, or normal force acting among the elements in contact, $f_{n}$. For case A, before the fracture, stresses uniformly increase 
(b)

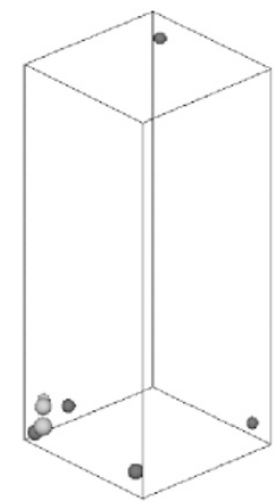

$e=0.00494$

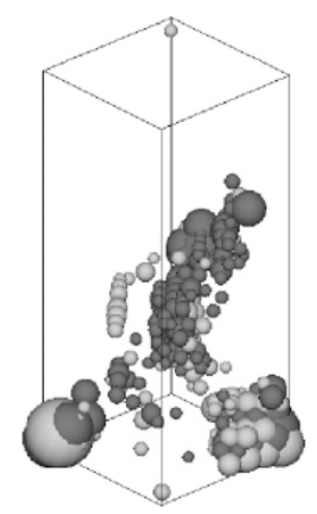

$e=0.00502$
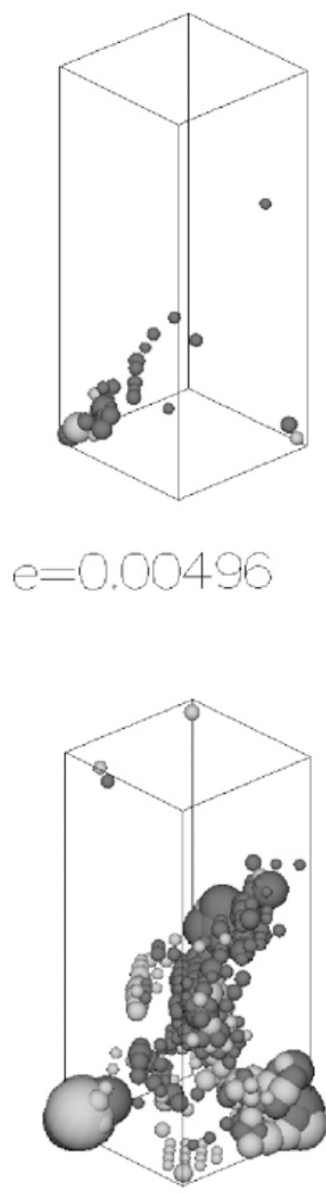

$e=0.00504$
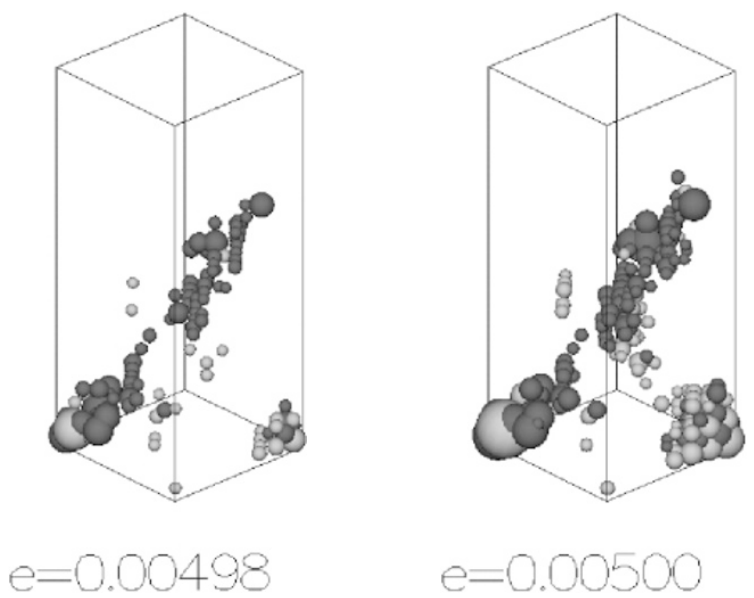

$e=0.00500$
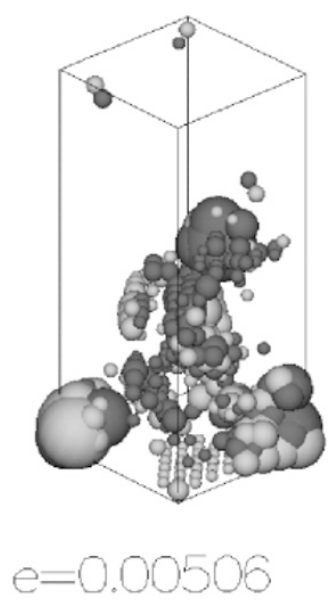

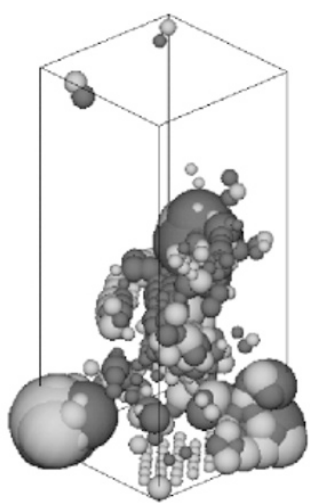

$e=0.00508$

Fig. 6. (continued).

in proportion to the strain $(e<0.00300)$, indicating elastic deformation. However, the stress concentrates gradually on the edges of model rock sample, especially the corners ( $e \sim 0.00498)$. As we assume the friction on the top and bottom surfaces, radial compression stresses develop from near surfaces of both sides in a similar way to deformation experiments (Vutukuri et al., 1974). When the cracking occurs, the spheres first disappear, i.e., lose the connection, at the corners, and then, the release of normal stress propagates to inside of the rock ( $e \sim 0.00540-0.00581)$, with simultaneous propagation of cracking, that is, fracturing. Areas of stress accumulation are almost coincident with the areas of stress concentration predicted by the Griffith theory (Hawkes and Mellor, 1970). After the instantaneous crack propagation, the stress is almost completely mitigated ( $e \sim 0.00590)$. For case $\mathrm{B}$, the inner stress increases inside the rock similar to that in case A until $e \sim 0.00300$. After $e \sim 0.00490$, the stress concentrates in some parts of the rock sample. However, fracturing occurs from the surface and propagates into the inner part of the sample. The stress accumulation is found near the fracturing surface after fracture, at least till $e \sim 0.00600$.

Figures 6(a) and (b) illustrate the increment of the rota- tion angle of elements. The size of spheres is proportional to the rotation angle around the $x$-axis and the colors indicate the direction (clockwise; light gray and counterclockwise; dark gray). Elements rotate largely near the corner and adjoining elements rotate in the opposite direction each other. The rotation of elements tends to be larger on the fracturing surfaces, especially for case B (Fig. 6(b)). The largest rotation angle becomes 20-30 degrees during stress drop. After breaking connections, the elements are allowed to rotate freely and a part of elastic energy may be transferred into rotation energy of elements. These free rotatable elements may correspond to the gouge and their rotation results in movement of the rock units, i.e., faulting, accompanying the fracture, smoother (Scott, 1996).

Figures 7(a) and (b) show the relationship between normal $\left(f_{n}\right)$ and tangential $\left(f_{s}\right)$ forces (proportional to stresses) between elements. Coulomb-Mohr's failure criterion (Eq. (12)) is also shown by broken lines. Before fracturing, both the normal and shear stresses concentrate on narrow range for both cases. As the fracturing proceeds, the stress values become dispersed. After that, the stresses of both components decrease with time. After cracking, since the stress is controlled by the friction between elements (Eq. (14)), a lin- 

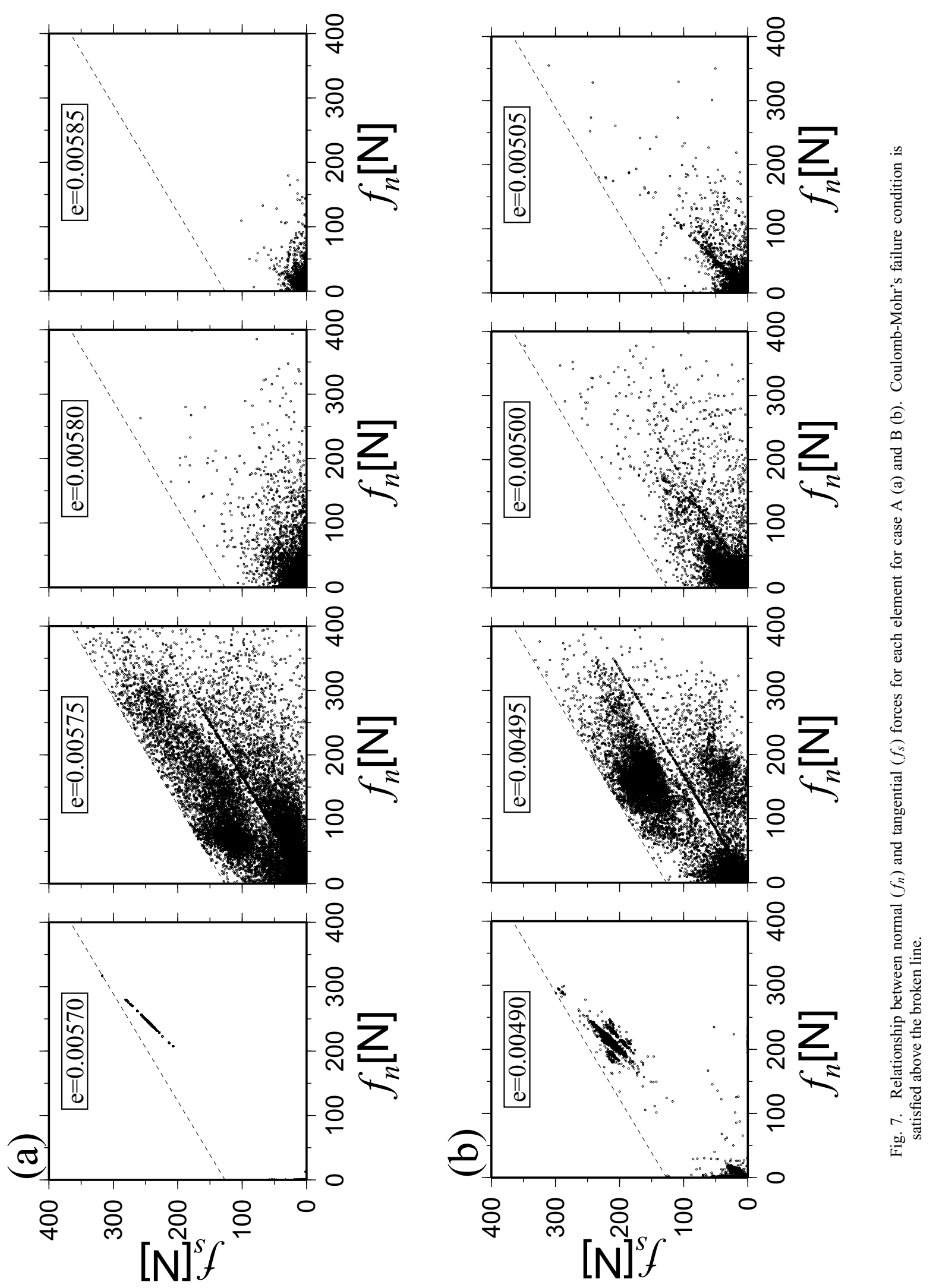


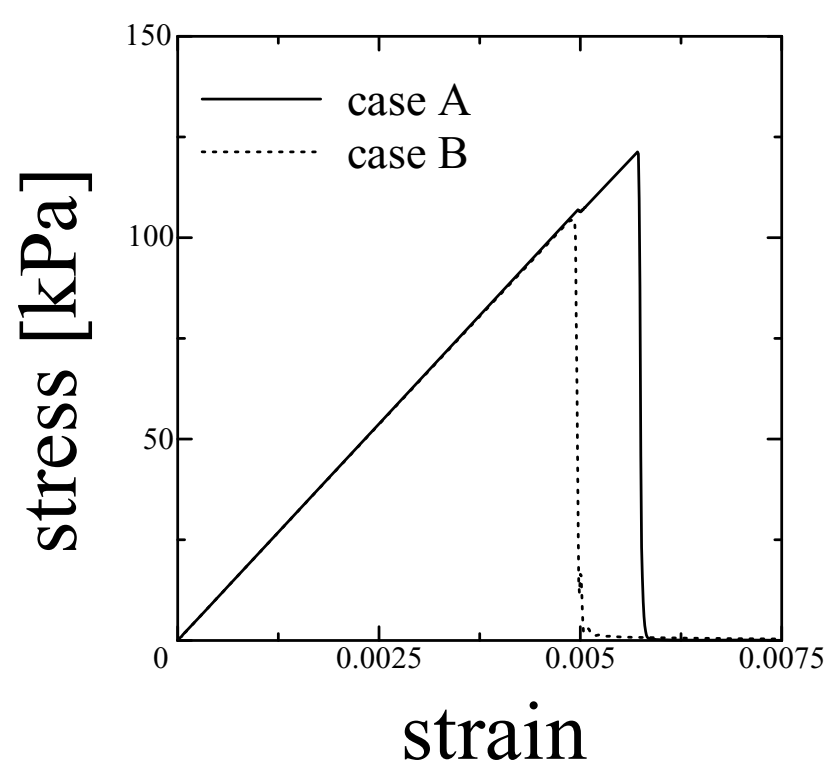

Fig. 8. Stress-strain curves for cases A (solid line) and B (broken line). The vertical stress on the top surface (vertical axis) and the strain along axis (horizontal axis) are shown.

ear relationship between $f_{n}$ and $f_{s}$ can be seen. This trend is weak for case A compared with case B. This can be explained by the fact that the flat fracturing surface in case B is smoother than the cone-shaped fracturing surface in case A in this condition (see, Fig. 4(b)). The result of case A suggests that the roughness of fracturing surface may act as fault asperity (Scholz, 1990) and may cause concentration of normal stress after fracturing (see, also Fig. 5(a)).

\subsection{Stress-strain relationship}

Figure 8 shows the relationship between axial strain and stress for cases A and B. All compression axes are in the vertical direction at the ends of model rock sample. The axial stress is calculated from dividing the summation of the force of each element by the upper surface area. A stress drop occurs at $e \sim 0.00572$ for case A and $e \sim 0.00492$ for case B. The stress-strain curves show abrupt transitions from the elastic regime to the brittle regime during the fracturing process, which is generally observed in the short term uniaxial compression tests in the laboratory (e.g., Wawersik and Brace, 1971). The peak stresses, which indicate the strength of whole rock, are an order of $100 \mathrm{kPa}$ for both cases. Young's moduli of rocks forming the crust and upper mantle $\left(\sim 10^{9} \sim 10^{10} \mathrm{~kg} \mathrm{~m}^{-1} \mathrm{~s}^{-2}\right)$ are 100-1000 times larger than that used in this study $\left(\sim 10^{7} \mathrm{~kg} \mathrm{~m}^{-1} \mathrm{~s}^{-2}\right)$. Thus, the peak stresses in our results $\left(\sim 10^{5} \mathrm{~Pa}\right)$ would be about $1 / 1000$ of those for natural rocks $\left(\sim 10^{8} \mathrm{~Pa}\right)$ (Wawersik and Brace, 1971). The strength in case $B$ is about ten percents smaller than that in case A, although the averaged cohesion force per connection in case B is 0.5 percent smaller than that in case A. This result implies that the macroscopic fractures are determined by the interference and linkage of cracks generated at the weak part.

\section{Concluding Remarks}

In this study, rock fracturing is modeled on basis of the discrete element method, in which rock is assumed as an assemblage of spherical discrete elements obeying Newton's equations of motion of both translation and rotation. Cohesion force is newly considered between connected elements for treatment of continuum-discontinuum media. This method is useful to describe the fracturing due to the inherent discretization of model and can also treat the elastic bodies.

Numerical experiments of uniaxial compression of rocks using our model to study the brittle behavior show some interesting features. The cracking initiates at the edge(s) of rock sample and propagates inside. The surfaces of the fracture are cone-shaped for the rock with initially symmetric $f c c$ configuration (case A), and oblique if connections between the elements are randomly reduced (case B). The latter case, especially, shows the remarkable localization of deformation along the shear zone. The stress distribution inside the rock and the rotation of elements are also demonstrated. The existence of defective connections yields the localization of the stress. Negative correlation between the inner stress and rotation of elements is found. The stress-strain curves also show a good agreement with those observed in experimental uniaxial compression tests (e.g., Wawersik and Brace, 1971). After the instantaneous crack propagation, the stress inside the rock is almost completely mitigated by the fracturing. The results in our model is also consistent with theoretical works of the rock fracture (e.g., Hawkes and Mellor, 1970; Bordia, 1971). The patterns of the surface of the fracture show an agreement with those observed in the laboratory experiments (e.g., Lama, 1966; Vutukuri et al., 1974; Paterson, 1978), though their feature changes by introducing only one percent weak connections inside the rock. This results suggest that the fracture pattern is controlled by the heterogeneity of strength within rock.

In the present model, number of elements is at least $\sim 4 \times 10^{3}$ and the Young's modulus is smaller than those of the natural rocks by the limitation of computer facilities. As the movements of elements given by uniaxial compression $\left(\sim 10^{-3} \mathrm{~m} \mathrm{~s}^{-1}\right)$ are much smaller compared to the propagation velocity of cracks, the qualitative results including fracture pattern would be valid to the natural rocks. However, the use of the parameters comparable to the natural rocks and increase in the number of elements will be necessary for further quantitative investigation. Besides, we may need to adopt the variation of the radii of elements for smoothing the surface of fracture. Thermal effects should be also incorporated, if the scheme is applied to larger scale simulations such as the tectonic models.

Acknowledgments. We are grateful to Prof. S. Honda and two anonymous reviewers for insightful comments and a better perspective of our work. The comments of Drs. N. Suda and T. Nakakuki are most helpful. We also thank Ms. R. Yokochi and an anonymous reviewer for correcting the English which improved the manuscript. Runs of this research were mainly made on computers of Institute for Nonlinear Sciences and Applied Mathematics (INSAM) in Hiroshima University.

\section{References}

Bird, P. and X. Kong, Computer simulations of California tectonics confirm very low strength of major faults, Geol. Soc. Am. Bull., 106, 159-174, 
1994.

Bordia, S. K., The effects of size and stress concentration on the dilatancy and fracture of rock, Int. J. Rock Mech. Min. Sci., 8, 629-640, 1971.

Cundall, P. A. and O. D. L. Strack, A discrete numerical model for granular assemblies, Géotechnique, 29, 47-65, 1979.

Donzé, F., P. Mora, and S. A. Magnier, Numerical simulation of faults and shear zones, Geophys. J. Int., 116, 46-52, 1994.

Hawkes, I. and M. Mellor, Uniaxial testing in rock mechanics laboratories, Eng. Geol., 4, 177-285, 1970.

Holland, D. and M. Marder, Ideal brittle fracture of silicon studied with molecular dynamics, Phys. Rev. Lett., 80, 746-749, 1998.

Iwashita, K. and M. Oda, Rolling resistance at contacts in simulation of shear band development by DEM, J. Engrg. Mech., ASCE 124(3), 285292, 1998.

Knott, J. F., Fundamentals of Fracture Mechanics, 273 pp., Butterworth, London, 1973.

Lama, R. D., Elasticity and strength of coal seams in situ and an attempt to determine the energy in pressure bursting of roadsides, D. Sc. Tech. Thesis, Faculty of Mining, Academy of Min. \& Metall., Cracow, Poland, 1966.

Mora, P. and D. Place, Numerical simulation of earthquake faults with gouge: toward a comprehensive explanation for the heat flow paradox, J. Geophys. Res., 103(B9), 21067-21089, 1998.

Paterson, M. S., Experimental Rock Deformation-the Brittle Field,
254 pp., Springer-Verlag, Berlin, 1978.

Place, D. and P. Mora, The lattice solid model to simulate the physics of rocks and earthquakes: Incorporation of friction, J. Comp. Phys., 150, 373-393, 1999.

Scholz, C. H., The Mechanics of Earthquakes and Faulting, 439 pp., Cambridge Univ. Press, New York, 1990.

Scott, D. R., Seismicity and stress rotation in a granular model of the brittle crust, Nature, 381, 592-595, 1996.

Tuckerman, M., B. J. Berne, and G. J. Martya, Reversible multiple time scale molecular dynamics, J. Chem. Phys., 97, 1990-2001, 1992.

Vutukuri, V. S., R. D. Lama, and S. S. Saluja, Handbook on Mechanical Properties of Rocks: Testing Techniques and Results, 300 pp., Trans Tech Publications, Clausthal, 1974.

Wawersik, W. R. and W. F. Brace, Post-failure behavior of a granite and a diabase, Rock Mech., 3, 61-85, 1971.

Weertman, J., Dislocation Based Fracture Mechanics, 524 pp., World Scientific, Singapore, 1996.

Wu, Z., A. Machida, and D. Gao, Development of mixed finite element method for composite discontinuous analysis, J. Geotech. Engrg., 598(I44), 149-159, 1998

Y. Matsuda (e-mail: yuya@geol.sci.hiroshima-u.ac.jp) and Y. Iwase 